INPLASY

PROTOCOL

To cite: Sun et al. A metaanalysis of plasma mercaptan levels in pregnant women with preeclampsia. Inplasy protocol 2021110065. doi:

10.37766/inplasy2021.11.0065

Received: 17 November 2021

Published: 17 November 2021

Corresponding author:

Hao Feng

szx7902@126.com

Author Affiliation:

Shandong First Medical

University

Support: None.

Review Stage at time of this submission: The review has not yet started.

Conflicts of interest:

None declared.

\section{A meta-analysis of plasma mercaptan levels in pregnant women with preeclampsia}

\author{
Sun, $\mathrm{X}^{1}$; Feng, $\mathrm{H}^{2}$; Lv, $\mathrm{X}^{3}$.
}

Review question / Objective: P Patients with preeclampsia and pregnant women with normal pregnancy; I Plasma thiol levels in patients with preeclampsia; C Plasma thiol levels in normal pregnant women; S RCT; $O$ Plasma thiol levels in patients with preeclampsia are lower than normal pregnant women.

Condition being studied: Plasma thiol levels in patients with preeclampsia.

Eligibility criteria: The review considered all published and unpublished randomised controlled trials (RCTs) evaluating the thoil level of patients with preeclampsia and normal pregnant women.

INPLASY registration number: This protocol was registered with the International Platform of Registered Systematic Review and Meta-Analysis Protocols (INPLASY) on 17 November 2021 and was last updated on 17 November 2021 (registration number INPLASY2021110065).

\section{INTRODUCTION}

Review question / Objective: P Patients with preeclampsia and pregnant women with normal pregnancy; I Plasma thiol levels in patients with preeclampsia; C Plasma thiol levels in normal pregnant women; S RCT; O Plasma thiol levels in patients with preeclampsia are lower than normal pregnant women.

Condition being studied: Plasma thiol levels in patients with preeclampsia.

\section{METHODS}

Participant or population: Patients with preeclampsia and pregnant women with normal pregnancy.

Intervention: Plasma thiol levels in patients with preeclampsia.

Comparator: Plasma thiol levels in normal pregnant women. 


\section{Study designs to be included: RCT.}

Eligibility criteria: The review considered all published and unpublished randomised controlled trials (RCTs) evaluating the thoil level of patients with preeclampsia and normal pregnant women.

Information sources: Cochrane, Embase, Web of science and PubMed database.

Main outcome(s): Compared with normal pregnant women, the plasma thiol content is significantly lower in patients.

Quality assessment / Risk of bias analysis: The risk of bias of each trial will be assessed by both authors, independently, using the Cochrane Collaboration Risk of Bias tool, with any disagreement resolved by consensus. The risk of bias tool comprises seven domains, including random sequence generation, allocation concealment, blinding of participants and personnel, blinding of outcome assessors, incomplete outcome data, selective outcome reporting, and other sources of bias . Reviewers will assign a judgement related to the risk of bias for each item, with a judgement of 'Yes' indicating low risk of bias, 'No' indicating high risk of bias and 'Unclear' indicating unclear or unknown risk of bias.

Strategy of data synthesis: Meta-analyses will be performed only if there are studies of similar comparisons reporting the same outcome measures. The authors combine risk ratios for dichotomous data, and mean differences for continuous data, using random-effects models and Review Manager (RevMan) 5.1 software. Heterogeneity was specifically examined with 12, and interpreted as follows (noting that the categories do overlap): $0-40 \%$ e might not be important; $30-60 \%$ e may represent moderate heterogeneity; $50-90 \%$ e may represent substantial heterogeneity, and $75-100 \%$ e represents considerable heterogeneity . If substantial or considerable heterogeneity was found, potential reasons for it were determined by conducting subgroup and sensitivity analyses where appropriate.
Subgroup analysis: If substantial or considerable heterogeneity was found, potential reasons for it will be determined by conducting subgroup and sensitivity analyses where appropriate.

Sensitivity analysis: If substantial or considerable heterogeneity was found, potential reasons for it will be determined by conducting subgroup and sensitivity analyses where appropriate.

Country(ies) involved: China.

Keywords: Hydrogen sulfide; Preeclampsia; Pregnancy; Meta-analysis.

Contributions of each author:

Author 1 - Zongxin Sun.

Author 2 - Hao Feng.

Author 3 - Xin Lv. 